\title{
Analysis and Optimization of Section-Thickness of the Manipulator for GIS Detection
}

\author{
Shuangyan $\mathrm{Su}^{1,2, \mathrm{a}}$, Yong Chang ${ }^{2}$ and Yonghe $\mathrm{Wei}^{1}$ \\ ${ }^{1}$ College of mechanical engineering, Shenyang Ligong University, 110159 Shenyang Liaoning, China \\ ${ }^{2}$ The State Key Laboratory of Robotics, Shenyang Institute of Automation, Chinese Academy of Sciences, 110016 Shenyang Liaoning, \\ China
}

\begin{abstract}
In order to find out the influence of different section-thickness of the manipulator for GIS detection on the mass, strength and stiffness, the performance of the arm rod with different section thickness is analysed by MATLAB under the condition of a certain section height. Firstly, the influence of the parameters of each section-thickness on the mass, strength and stiffness is analysed by single variable analysis. Then the mathematical model is established, and the section-thickness of the arm is optimized using the method of nonlinear programming. The results are similar to the results of single variable analysis. Finally, the section thickness of the virtual prototype is optimized by the finite element analysis method. The correctness of MATLAB optimization results is verified. The optimal solution of the section-thickness of the GIS-detection-arm is obtained.
\end{abstract}

\section{Introduction}

The X-ray digital imaging detection system, which is the detection equipment in electricity substation, has been successfully applied to the detection of GIS [1-2] (gas insulated switchgear) and other equipment. At present, we mainly depend on the way of manual lifting X-ray detection equipment to detect GIS. Because of the large volume and weight of X-ray detection equipment, the layout of the equipment is slow, the labour intensity of workers is great, and the risk in manual detection is high. The efficiency is quite low. Aiming at the abovementioned problems, the related groups development Xray non-destructive detection mobile manipulator. The corresponding arm, installed on the mobile platform, lift $\mathrm{X}$-ray detection device for GIS detection. As shown in Figure 1.

According to the requirement of better load capability and light weigh, the influence of different sectionthickness on the mass, strength and stiffness of the main linkage of the manipulator, installed on the mobile platform, is analysed. This paper uses three methods to optimize the section-thickness of the arm rod.

The optimization design of the section-thickness of the arm linkage for GIS detection is carried out on the basis of the initial mechanism and structure design. The total weight of manipulator is taken as the objective function and the mechanical performance parameters as considered as constraint condition. The decrease of the manipulator weight is realized, meanwhile the premise that the mechanical properties are within the limits.

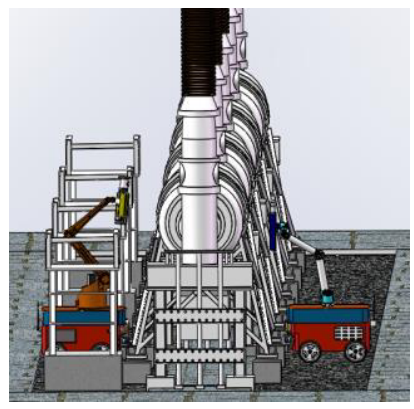

Figure1. GIS Detection

The research on the section shape and thickness has started from the end of 1980s. A parameter optimization method based on stiffness sensitivity analysis was proposed for the structural optimization of slender manipulator by Z. Luo and L. Liang et al in [3-4]. This work lays a theoretical foundation for the structural optimization for such manipulators. The structural and the control parameters of the whole arm system are optimized simultaneously using a genetic algorithm and the performance is compared with that of an arm with uniform links and an optimized control system by Y. Zhu, J. Qiu and T. Junji in [5]. The simulation result shows that faster regulation and less weight of the arm system can be achieved by simultaneous optimization. Stiffness model of space manipulator is established by $\mathrm{H}$. Jia et al in [6]. Optimal solution checking formula is derived by Lagrange number multiplication in this paper. The corresponding mechanical properties are verified by changing the thickness of the truss by Y. Yang in [7].

\footnotetext{
a Corresponding author: 18766562369@163.com
} 
According to the results, the analysis of the influence of the height of the cross section on the force is analysed.

For an attempt to resolve the problem in this paper, combined with the above mentioned methods, the three steps optimization principle for the optimization of section thickness of the GIS detection manipulator are proposed.

Step 1 Single variable analysis: Section-thickness value changed in turn, and its influence on the mass, strength and stiffness, get the proper ranges of each section-thickness value is analysed.

Step 2 Nonlinear programming method: Establish the objective function and the constraint conditions are defined firstly. Then the section-thickness is optimized by nonlinear programming method.

Step 3 Finite element analysis: Analyse the virtual prototype by the finite element analysis method is analysed. According to the results, the section-thickness is optimized by genetic algorithm.

Combined with three kinds of optimization results, the optimal solution of section thickness is obtained.

\section{Establishment of Mechanical Model}

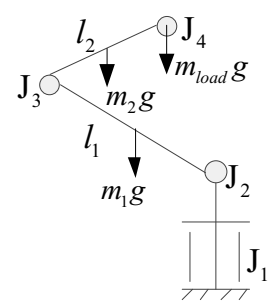

Figure 2. The Design Sketch of Manipulator Mechanism

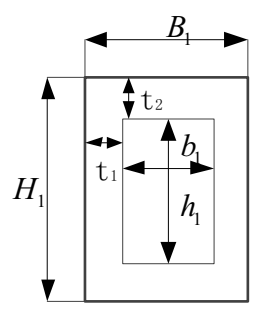

a)

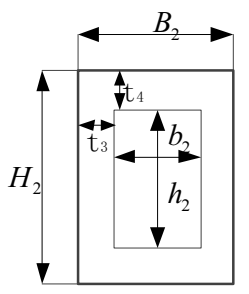

b)
Figure 3. The Cross-Section of Linkage

On the basis of the designed GIS detection manipulator, the mathematical model of the mechanics is established. The rod length of the two linkage are $l_{1}=1000 \mathrm{~mm}$ and $l_{2}=780 \mathrm{~mm}$, respectively. The load is about $30 \mathrm{~kg}$ in end. The cross section-height and cross section-width of the first linkage are $H_{1}=150 \mathrm{~mm}$ and $B_{1}=60 \mathrm{~mm}$, respectively. The cross section-height and cross sectionwidth of the second linkage are $\mathrm{H}_{2}=120 \mathrm{~mm}$ and $B_{2}=45 \mathrm{~mm}$, respectively. We take Super-duralumin 7075 as linkage material. Considering the mass and the force of the linkage, the safety factor is obtained according to the initial calculation moment, and the continuous torque of the most dangerous joint moment is $450 \mathrm{~N} \cdot \mathrm{m}$.

Figure 2 is the design sketch of manipulator mechanism. According to the actual working process, it is the most dangerous condition when the manipulator is in the fully folded state.

Figure 3 a) and b) are the cross section of the linkage 1 and 2 , respectively.

where $H_{i}(i=1,2)$ is the section-height of the arm rod 1 and $2, B_{i}(i=1,2)$ is the section- width of the arm rod 1 and 2 , respectively.

The masses of the two main linkage of the manipulator can be given by (1).

$$
\left\{\begin{array}{l}
m_{1}=0.81 t_{1}+0.324 t_{2}-0.0108 t_{1} t_{2} \\
m_{2}=0.505 t_{3}+0.18954 t_{4}-0.008 t_{3} t_{4}
\end{array}\right.
$$

where $m_{i}(i=1,2)$ is the mass of the arm rod, $t_{i}(i=1,3)$ is the side thickness, $t_{i}(i=2,4)$ is the thickness of top and down.

According to strength calculation formula of linkage $\sigma=\frac{M}{W_{z}}$, the normal stress of the two rod can be given by (2).

$$
\left\{\begin{array}{l}
\sigma_{1}=\frac{2.7 \times 10^{6}}{150 \times 60^{2}-\left(150-2 t_{2}\right)\left(60-2 t_{1}\right)^{2}} \\
\sigma_{2}=\frac{2.7 \times 10^{6}}{120 \times 45^{2}-\left(120-2 t_{4}\right)\left(45-2 t_{3}\right)^{2}}
\end{array}\right.
$$

where $\sigma_{i}$ is the stress of arm rod, $\omega_{i}$ is the deformation of arm rod, $M$ is the moment, and $W_{z}$ is the unbending section coefficient.

According to the principle of superposition of deflection in [8], the deformations of the two linkages are obtained. The stiffness model is established by (3).

$$
\left\{\begin{array}{l}
\omega_{1}=\frac{3.75 \times 10^{7}+1.736 \times 10^{5} m_{1}}{150 \times 60^{3}-\left(150-2 t_{2}\right)\left(60-2 t_{1}\right)^{3}} \\
\omega_{2}=\frac{2.9 \times 10^{4} m_{2}}{120 \times 45^{3}-\left(120-2 t_{4}\right)\left(45-2 t_{3}\right)^{3}}
\end{array}\right.
$$

\section{Single Variable Analysis}

\subsection{Relationship between section-thickness and mass}

Firstly, the influence of the section-thickness parameter of linkage 1 on the mass is analysed by MATLAB. The relationship between thickness $\left(t_{1}, t_{2}\right)$ and mass $\left(m_{1}\right)$ by $(1)$ is concluded, as shown in Figure 4.

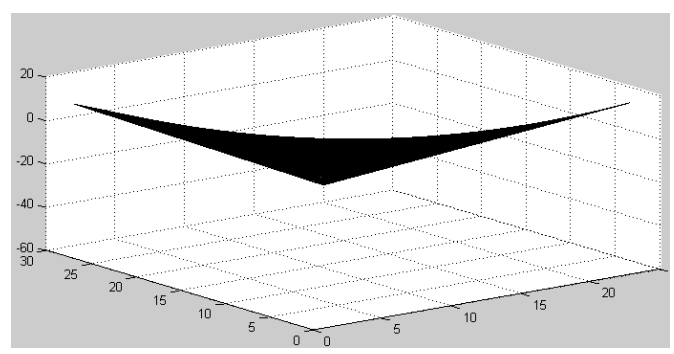

a) The relationship between $t_{1}, t_{2}$ and $m_{1}$ 


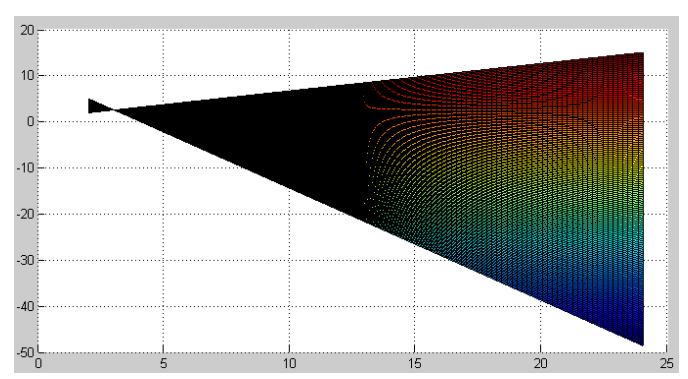

b) The relationship between $t_{1}$ and $m_{1}$

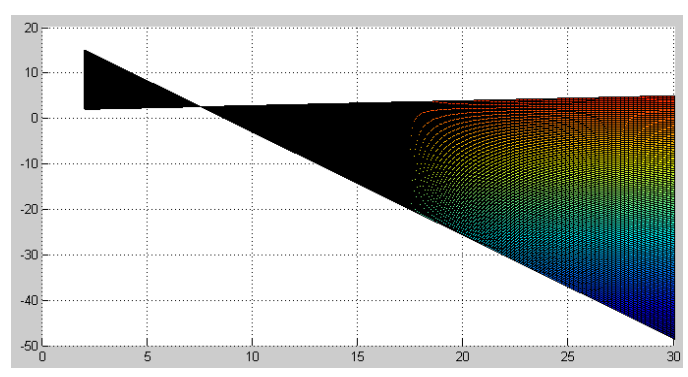

c) The relationship between $t_{2}$ and $m_{1}$

Figure 4. The Relationship between $t_{1}, t_{2}$ and $m_{1}$

From Figure 4, we can sum up that the mass variation range is not large when $t_{1} \geq 4 \mathrm{~mm}$ and the mass increases, but the range is not very large when $t_{2} \geq 8 \mathrm{~mm}$.

\subsection{Relationship between section-thickness and strength}

The following we analyze the effect of section-thickness variation on strength is analysed and the relationship between thickness $\left(t_{1}, t_{2}\right)$ and strength $\left(\sigma_{1}\right)$ by (2) is conclued, as shown in Figure5.

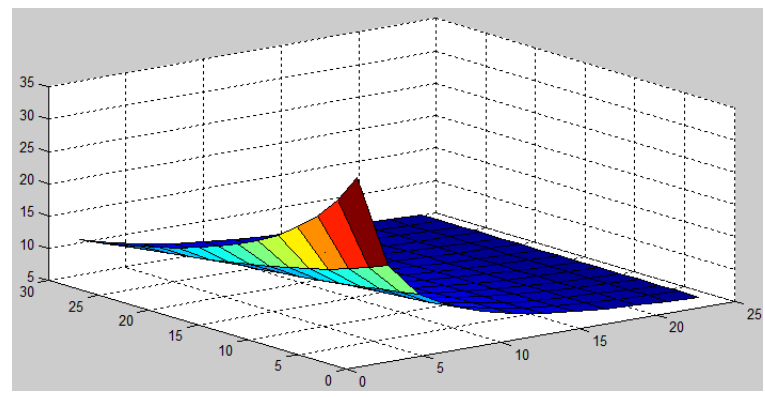

a) The relationship between thickness $\left(t_{1}, t_{2}\right)$ and strength $\left(\sigma_{1}\right)$

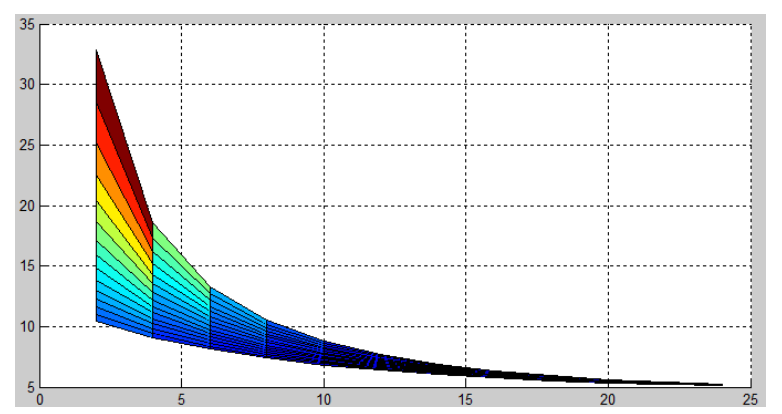

b) The relationship between thickness $\left(t_{1}\right)$ and strength $\left(\sigma_{1}\right)$

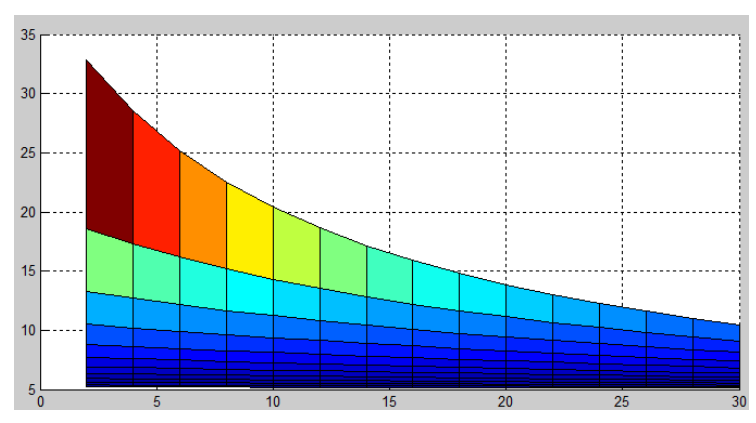

c) The relationship between thickness $\left(t_{2}\right)$ and strength $\left(\sigma_{1}\right)$

Figure 5. the Relationship between Thickness $\left(t_{1}, t_{2}\right)$ and Strength $\left(\sigma_{1}\right)$

Aluminum has good characteristics of stress. We can see from Figure 5 that when the thickness $t_{1} \geq 2 \mathrm{~mm}$, $\sigma_{1} \leq 33 \mathrm{MPa}$ and $t_{2} \geq 2 \mathrm{~mm}, \sigma_{1} \leq 33 \mathrm{MPa}$. We can sum up that the thickness can be easily satisfied in terms of stress.

\subsection{The relationship between section-thickness and stiffness}

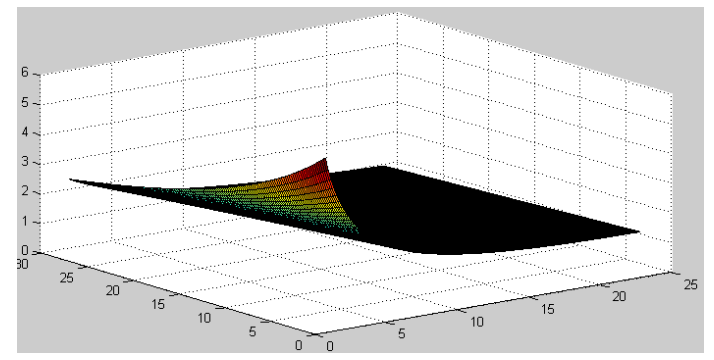

a) The relationship between thickness $\left(t_{1}, t_{2}\right)$ and stiffness $\left(\omega_{1}\right)$

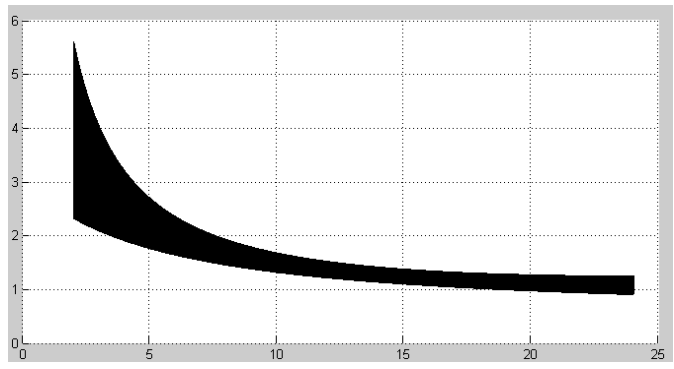

b) The relationship between thickness $\left(t_{1}\right)$ and stiffness $\left(\omega_{1}\right)$

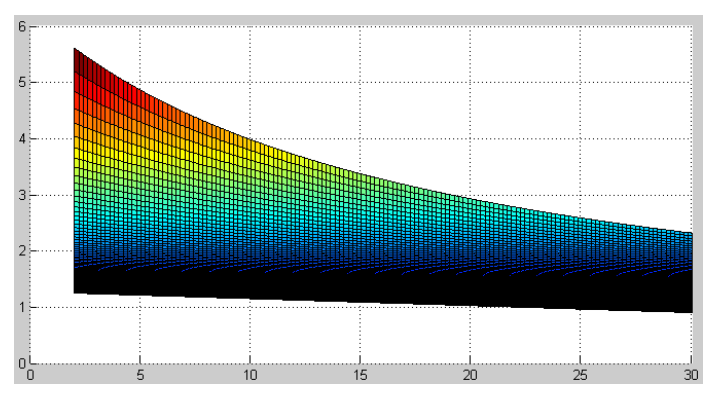

c) The relationship between thickness $\left(t_{2}\right)$ and stiffness $\left(\omega_{1}\right)$ Figure 6. The Relationship between Thickness $\left(t_{1}, t_{2}\right)$ and Stiffness $\left(\omega_{1}\right)$ 
The effect of section-thickness variation on stiffness is simulated and the relationship between thickness $\left(t_{1}, t_{2}\right)$ and stiffness $\left(\omega_{1}\right)$ by (3) is described, as shown in Figure 6.

We can see from Figure 6 that when the thickness $t_{1} \geq 5 \mathrm{~mm}, \omega \leq 5 \mathrm{~mm}$ and $t_{2} \geq 8 \mathrm{~mm}, \omega \leq 4 \mathrm{~mm}$.

Combined with the above three kinds of analysis results, it can be concluded that the best section-thickness of the linkage 1 is $t_{1}=4 \mathrm{~mm}$ and $t_{2}=8 \mathrm{~mm}$.

Through the above analysis method, in the same way, it can be concluded that the optimum section-thickness of the linkage 2 is $t_{3}=3 \mathrm{~mm}$ and $t_{4}=6 \mathrm{~mm}$.

\section{Nonlinear Programming}

The effect of the thickness on the mass, strength and stiffness of the single variable is analysed, and the optimal solution is obtained.

According to the constraint conditions and objective function, the mathematical model of nonlinear programming is established [9-10]. The comprehensive analysis of the effects of two thickness variables on mass, strength and stiffness is carried out.

The nonlinear programming model is as follows: $\min m_{i}(i=1,2)$

$$
\text { s.t. }\left\{\begin{array}{l}
\frac{M_{1}}{W_{z 1}}=\sigma_{1} \leq[\sigma] \\
\omega_{1}=\frac{M_{1} l_{1}^{3}}{2 E I}-\frac{m_{1} g\left(l_{1} / 2\right)}{6 E I}\left(3 l_{1}-\frac{l_{1}}{2}\right) \leq[\omega] \\
\frac{M_{2}}{W_{z 2}}=\sigma_{2} \leq[\sigma] \\
\omega_{2}=\frac{m_{2} g\left(l_{2} / 2\right)}{6 E I}\left(3 l_{2}-\frac{l_{2}}{2}\right)+\frac{m_{2} g l_{2}^{2}}{3 E I} \leq[\omega] \\
t_{\mathrm{i}, \min } \leq t_{i} \leq t_{\mathrm{i}, \max }(\mathrm{i}=1,3) \\
t_{1,3} \leq \frac{B_{1,2}}{2} \\
t_{\mathrm{i}, \min } \leq t_{i} \leq t_{\mathrm{i}, \max }(i=2,4) \\
t_{2,4} \leq \frac{H_{1,2}}{2}
\end{array}\right.
$$

where $E$ is elastic modulus of Aluminium alloy and $I$ is moment of inertia of cross section.

Based on the above model, the section-thickness is optimized by the nonlinear programming method using fmincon function in MATLAB optimization toolbox. The results of $t_{1}, t_{2}, t_{3}, t_{4}$ are as follows:

\section{$\begin{array}{llll}8.6814 & 3.5183 & 5.9878 & 3.4042\end{array}$}

The masses of the two linkages are as follows, respectively.

\section{$7.8420 \quad 3.5000$}

The optimization results difference between the nonlinear programming method and single variable analysis is very small.

\section{Optimization of Finite Element Analysis}

We model according to the designed parameters, as shown in Figure7.

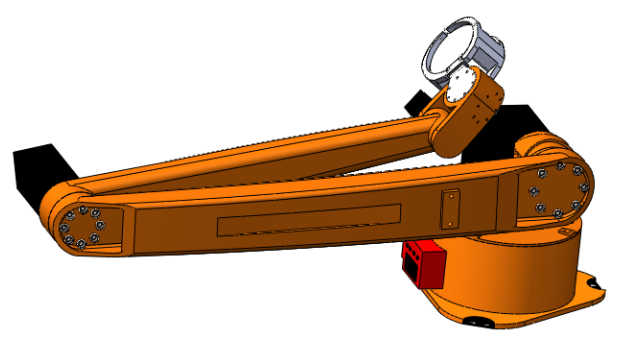

Figure 7. Model of Manipulator

The initial section-thickness values of the linkage 1 are $t_{1}=7 \mathrm{~mm}$ and $t_{2}=12 \mathrm{~mm}$. The mass is $10 \mathrm{~kg}$. The results of finite element analysis of the rod 1 are as shown in Figure8:

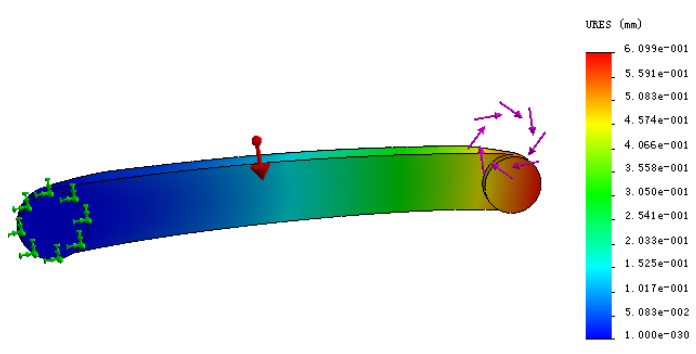

a) The analysis result of displacement

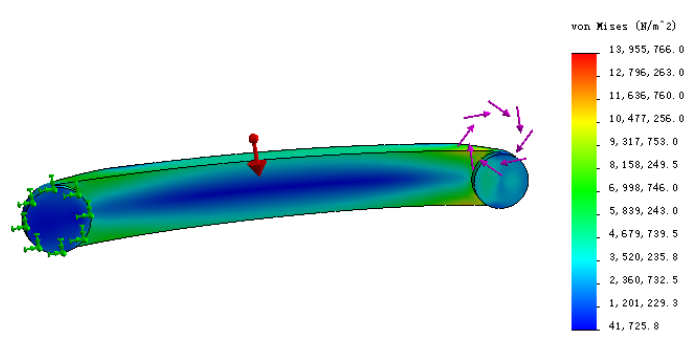

b) The analysis result of stress

Figure 8. The Results of Finite Element Analysis

Using finite element analysis [11] and genetic algorithm, we take the section thickness of $t_{1}$ and $t_{2}$ as design variables, take the stress and deformation as the constraint condition, and take mass as the optimization goal, to optimize the model. The optimization results of rod 1 and 2 are as shown in Table 1:

Table 1. The optimization results of rod 1 and 2

\begin{tabular}{|c|c|c|}
\hline & Rod 1 & Rod 2 \\
\hline$t_{1,3}$ & $8 \mathrm{~mm}$ & $3 \mathrm{~mm}$ \\
\hline$t_{2,4}$ & $4 \mathrm{~mm}$ & $3.5 \mathrm{~mm}$ \\
\hline Stress & $21.393 \mathrm{MPa}$ & $17.349 \mathrm{MPa}$ \\
\hline Deformation & $0.88929 \mathrm{~mm}$ & $0.00053 \mathrm{~mm}$ \\
\hline Mass & $7.035 \mathrm{~kg}$ & $3.011 \mathrm{~kg}$ \\
\hline
\end{tabular}




\section{Conclusions}

According to the structure of the typical example of GIS detection manipulator, the mathematical model is established. Analysis and optimization of the mass, stiffness and strength of the manipulator are carried out. The optimal solution is obtained through a single variable analysis. Then, the top and down section thickness and the side thickness of the linkage are optimized by the method of nonlinear programming. Finally, the demension of section is optimized by the finite element method. Through three different analysis optimization methods, the optimal solution of section thickness is obtained.

The optimization results show that the weight of the manipulator decreases obviously according to the decrease of the thickness, and the stress and deformation increases slightly. The work of this paper used as the preliminary research for designation of the manipulator in detail.

\section{References}

1. X. Zheng, J. Chen. New techniques for fault detection in GIS [J]. Guangdong electric power, 2001, 14 (5): 43-45.

2. http://baike.baidu.com/link?url=vt08j5CBKBdgz8hb pj8V5yqOF4IvHWKM4VeEdyzfxdLI1JvbRCsRLW mhTCJJrSJ8RWt9uPVxK9CTM6VXj8YweK
3. Z. Luo, L. Liang, Y. Chen, et al. Sensitivity analysis and parameter optimization of the stiffness of slender manipulator [J]. Journal of Northeastern University: Natural Science Edition, 2011, 32 (9): 1319-1323.

4. Shen J, Yoon D. A new scheme for efficient and direct shape optimization of complex structures represented by polygonal meshes[J]. International Journal for Numerical Methods in Engineering, 2003, 58(14):2201-2223.

5. Ye Z, Qiu J, Junji T. Simultaneous optimization of a two-link flexible robot arm[J]. Journal of Robotic Systems, 2001, 18(1):29-38.

6. H. Jia, Q. Yao, Q. Huang. Stiffness optimization of space manipulator based on mass distribution [J]. China Space Science and technology, 2008 (3): 4552.

7. Y. Yang, X. Liang. Analysis of the influence of the height of the cross section of the arm on the strength of the rod [J]. construction machinery, 1994 (9): 2427.

8. H. Liu. Advanced material mechanics $[\mathrm{M}]$. higher education, 1985.

9. D. Xue. MATLAB solution to the problem of higher applied mathematics $[\mathrm{M}]$. Tsinghua University press, 2008.

10. J. Chen. MATLAB collection $[\mathrm{M}]$. Electronic Industry Press, 2011.

11. H. Zhang. ANSYS Workbench14.5 numerical simulation engineering case analysis $[\mathrm{M}]$. Machinery Industry Press, 2013. 